\title{
RETROSPECTIVE ANALYSIS OF FOLLICULAR LYMPHOMA PATIENTS IN TRAKYA UNIVERSITY SCHOOL OF MEDICINE: A SINGLE CENTER EXPERIENCE
}

\author{
Fatih Erkan Akay $^{1}$ (D), Beliz Koçyiğit ${ }^{1}$ (D), Fevzi Oktay Şişman ${ }^{1}$ (D), Mert Yücel Ayrık ${ }^{1}$ (D) İpek Savaşır $^{1}$ (D), \\ Elif Cengiz ${ }^{1}$ (D), Ufuk Demirci ${ }^{2}$ (D), Hakkı Onur Kırkızlar² (D)
}

${ }^{1}$ Trakya University School of Medicine, Edirne, TURKEY

${ }^{2}$ Division of Hematology, Department of Internal Medicine, Trakya University School of Medicine, Edirne, TURKEY

\begin{abstract}
Aims: To establish a dataset including demographic features, disease characteristics, and survival rate of follicular lymphoma patients in Trakya University School of Medicine and contribute to the database of follicular lymphoma in Turkey. Methods: In this retrospective cross-sectional study, we analyzed data constituting of follicular lymphoma patients over 18 years of age followed during the years of 2015-2020 in Trakya University Division of Hematology. Results: Out of 43 patients, $22(51.2 \%)$ were female and 21 (48.8\%) were male. The mean age was 56.56 (standard deviation 13.24) years. There were 5 (11.6\%) patients with B symptoms, presence of bone marrow involvement was seen in 17 (39.5\%) patients, lastly, there were 18 (41.9\%) patients with splenomegaly. Twenty-one (48.8\%) patients received rituximab, cyclophosphamide, doxorubicin hydrochloride, vincristine sulfate, and prednisone, making it the most common treatment protocol administered in our study. Conclusion: Follicular lymphoma patients usually end up getting diagnosed at an advanced stage of the disease, presenting with incidentally noticed painless lymphadenopathy. Additionally, based on evidence in the literature, a clear gap in the successful diagnosis of follicular lymphoma patients can be observed between developed and developing countries. To overcome this hurdle, enhanced cooperation with hematopathology may lead to an increased awareness enabling physicians to make a more accurate diagnosis. Nonetheless, further studies are still needed to fully apprehend the epidemiology of follicular lymphoma patients in Turkey. Keywords: Follicular lymphoma, retrospective study, non-Hodgkin's lymphomas
\end{abstract}

\section{INTRODUCTION}

Representing more than $20 \%$ of all non-Hodgkin's lymphomas (NHLs), follicular lymphoma (FL) is the most common indolent systemic lymphoid neoplasm originating from germinal core $\mathrm{B}$ cells, both centrocytes, and centroblasts, usually exhibiting a follicular growth pattern (1-3). Having a higher incidence (7/100.000) in western countries, it primarily occurs in adults, mean age of 65 , with a male-to-female ratio of 1: 1.7 (4). An increased risk factor in the growth of tumor has been linked in patients who had higher exposure to herbicides and pesticides (5).

Most FL patients exhibit a widespread disease at diagnosis, characterized by generalized painless lymphadenopathy, splenomegaly, and bone marrow involvement, whereas extranodal involvement is less common. B symptoms (weight loss, fever, and night sweats) are usually seen in $20 \%$ of the patients (6). Change in clinical course, rapid enlargement of a mass, $\mathrm{B}$ symptoms, and abnormal laboratory findings (elevated serum lactate dehydrogenase (LDH)) could indicate transformation to a more aggressive type of non-Hodgkin's lymphoma (NHL), for example, diffuse large B cell lymphoma (a phenomenon also known as Richter's syndrome) $(2,6)$.

The patient's evaluation should be made through his history, laboratory findings, physical examination, and imaging. Diagnosis is made with histopathological findings of an excisional lymph node biopsy. More than $85 \%$ of cases have a characteristic (14:18) translocation - a fusion between the BCL-2 gene on chromosome 18 and the IgH locus on chromosome 14 (7).

Histopathologically, World Health Organization (WHO) classifies FL into 3 grades according to the number of centroblasts (large unnotched follicle cells) spotted per high-power field (2). As for disease staging, the Ann Arbor system plays a crucial role together with modified Groupe d'Etude des Lymphomes Folliculaires (GELF) or British National Lymphoma Investigation criteria in determining the choice of treatment (8). Furthermore, prognostic tools established before the rituximab era, so-called FL International Prognostic Index (FLIPI) and FLIPI-2, are frequently used for risk assessment (8). Development into a more aggressive phenotype is observed at 5 years in $11-13 \%$, and at 10 years in $15-28 \%$ of the patients $(1,6)$.

Disease management strategies include a watch-and-wait approach, immunochemotherapy, R-CHOP (rituximab, cyclophosphamide, doxorubicin, vincristine, prednisone), single-agent therapy, and radiotherapy $(3,9)$. In terms of progression-free survival, favorable outcomes are noted in watch-and-wait and single-agent therapy options. Bone marrow transplantation is the preferred management in patients with a relapsed/refractory clinical course or Richter's syndrome. Thus, a better understanding of the tumor's genetic map and microenvironment may provide hope for novel targeted therapies in FL patients. 
This retrospective study aims to establish data; including demographic features, disease characteristics, and total follow-up of FL patients in Trakya University School of Medicine and contribute to the database of FL patients in Turkey.

\section{MATERIAL AND METHODS}

This retrospective study was approved by the Scientific Research Ethics Committee of Trakya University School of Medicine (Protocol Code: TÜTF-BAEK 2020/339). Analyzed data constituted FL patients over 18 years of age followed during the years of 2015-2020 in Hematology Division, Trakya University School of Medicine. Patients' data compromised their age, gender, date of diagnosis, last inspection date, neutrophil count, LDH levels, Ann Arbor scores, lymph node involvement, bone marrow involvement, serum $\beta 2$ microglobulin levels, the existence of B symptoms, and treatment method initiated.

Numbers, percentages, mean and standard deviation (SD) were used as the descriptive statistics. The variables were tested for normal distribution by the Shapiro-Wilk test. Normal distribution was observed in age, lymph node involvement number, neutrophil count, and platelet count, thus they were presented as mean \pm SD. Non-normal distribution was observed in gender, duration of total follow-up, hemoglobin levels, serum $\beta 2$ microglobulin, and LDH levels, which were presented as median and inter-quartile range (IQR). WHO classification, Ann Arbor stage, FLIPI-1, and FLIPI-2 scores, GELF criteria, B symptoms, bone marrow involvement, splenomegaly and treatments were presented as numbers and percentages. Kaplan-Meier analysis was used to demonstrate patients' total follow-up. A p-value $<0.05$ was set for statistical significance. The data were analyzed with IBM SPSS version 23.0.

\section{RESULTS}

Out of 43 FL patients, $22(51.2 \%)$ of them were female and 21 (48.8\%) of them were male. The mean (SD) age was 56.56 (13.24) years. There were $5(11.6 \%)$ patients with B symptoms, presence of bone marrow (BM) involvement was seen in 17 (39.5\%) patients, and lastly, there were 18 (41.9\%) patients with splenomegaly. The summary statistics for the demographic data and total follow-up of FLIPI scores of patients are presented in Table 1 and Figure 1.

According to the WHO classification for FL, there were 9 (20.9\%) grade 1, $17(39.5 \%)$ grade 2, $3(7.0 \%)$ grade 3, $6(14.0 \%)$ grade $3 \mathrm{~A}, 1(2.3 \%)$ grade $3 \mathrm{AB}$, and $5(11.6 \%)$ grade $3 \mathrm{~B}$ patients, and $2(4.7 \%)$ with missing data. According to the Ann Arbor staging system, there were $5(11.6 \%)$ patients in stages I-II, $31(72.1 \%)$ patients in III-IV, and 7 (16.3\%) missing data. Based on GELF criteria, there were $16(37.2 \%)$ patients with 0 points, $8(18.6 \%)$ patients with 1 point, $11(25.6 \%)$ patients with 2 points, 7 (16.3\%) patients with 3 points, and $1(2.3 \%)$ patient with 4 points.

Summary statistics for numerical data of treatment methods applied to patients are presented in Table 2. There were $3(7.0 \%)$ patients with Richter's syndrome, and 2 (4.7\%) patients with skin involvement. The mean \pm SD for number of lymp nodes involved was $7.43 \pm 5.28$, for the neutrophil count was $4384 \pm 1858 \mathrm{~mm} 3$, and for the platelet count was $230.74 \pm 93.44 \mathrm{~mm} 3$. Median (IQR) for hemoglobin was 13.30 (3) g/dL, for lactate dehydrogenase was 210.00 (182) U/L, and for serum $\beta 2$-microglobulin was 2408 (2796) $\mathrm{mcg} / \mathrm{mL}$.
Table 1: Summary statistics for demographic data, total follow-up of FLIPI scores of patients.

Number of patients $(n=43)$

\begin{tabular}{ll}
\hline Gender [n(\%)] & $22(51.2)$ \\
Female & $21(48.8)$ \\
Male & $56.53 \pm 13.24$ \\
Age of the patients during diagnosis (years) \\
Total follow-up (months) \\
FLIPI-1 score [n (\%)] \\
Low-Risk (0-1) \\
Intermediate-Risk (2) \\
High-Risk ( $\geq 3)$ \\
FLIPI-2 score [n (\%)] & $13(30.2)$ \\
Low-Risk (0-1) & $14(37.2)$ \\
Intermediate-Risk (2) & $22(51.2)$ \\
High-Risk ( $\geq 3)$ & $7(16.3)$ \\
\hline FLIPI: Follicular Lymphoma International Prognostic Index
\end{tabular}

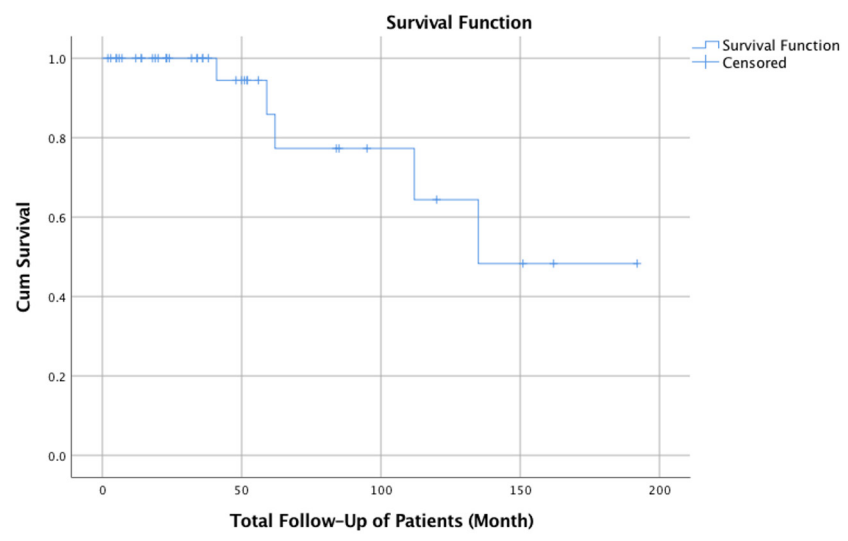

Figure 1: Kaplan-Meier survival analysis of patients' total follow-up.

Table 2: Summary statistics for treatment protocols administered.

\begin{tabular}{lll} 
Treatment Protocol & $\begin{array}{l}\mathbf{1}^{\text {st }} \text { Line } \\
\text { Treatment N(\%) }\end{array}$ & $\begin{array}{l}\geq 2^{\text {nd }} \text { Line Treat- } \\
\text { ment } N(\%)\end{array}$ \\
\hline $\begin{array}{l}\text { Watch and Wait } \\
\text { R-CHOP }\end{array}$ & $1(20.9)$ & \\
R-CVP & $23(53.5)$ & $1(2.3)$ \\
R-ICE & $9(20.9)$ & $2(4.7)$ \\
R-BENDA & & \\
R & $3(7.0)$ & $1(2.3)$ \\
R-CEOP & $9(20.9)$ & \\
$\begin{array}{l}\text { Obinutuzumab, Lenalidomide } \\
\text { and Bendamustine }\end{array}$ & $1(2.3)$ & $1(2.3)$ \\
Radiotherapy & $1(2.3)$ & $1(2.3)$ \\
\hline
\end{tabular}

N: Number, R-CHOP: Rituximab, cyclophosphamide, doxorubicin hydrochloride, vincristine sulfate, and prednisone, R-CVP: Rituximab, cyclophosphamide, vincristine sulfate, and prednisone, R-ICE: Rituximab, ifosfamide, carboplatin, and etoposide phosphate, R-BENDA: rituximab and bendamustine, R: Rituximab, R-CEOP: Rituximab, cyclophosphamide, etoposide, vincristine, prednisone 


\section{DISCUSSION}

The primary objective of this retrospective study was to analyze FL patients' demographic features, disease characteristics, overall survival and treatment methods followed during the years of 20152020 in the Hematology Division of Trakya University School of Medicine.

The mean age in our study was 56.56 years, slightly lower compared to the results of Becnel et al. (10), which was between 60-70 years of age. However, results in the study conducted by Federico et al. (11) were in line with ours, regarding age and gender demographics. In our study, $51.2 \%$ of the patients were female and $48.8 \%$ were male, whereas their patient percentages were $53 \%$ female and $47 \%$ male. The difference between the two studies was the patient population, Federico et al. (11) studied 987 patients, whereas 43 patients were studied in ours. A similar database to ours, Kahraman et al. (12) had 17 female (60.7\%) and 11 male (39.3\%) patients, however, $50 \%$ (12) of their patients were younger than 60 years, and the other half were older.

Observing the overall number of lymphoma patients followed in our hospital between the years of 2015-2020, there were 87 Hodgkin's lymphoma and 566 NHL patients, of which 43 (7.6\%) were FL patients (unpublished data, personal communication with Kirkizlar et al.). Our results were collateral with a similar study carried out in Turkey by Kahraman et al. (12) suggesting that FL can be seen in $9 \%$ of all NHLs in Turkey. On the other hand, Carbone et al. (13) reported that FL may represent up to $20-25 \%$ of NHL in developed countries, based on evidence reviewed, a clear gap between patients in developed and developing countries is discernible when it comes to diagnosis of FL. This gap is may be attributed to the lack of resources physicians have in order to produce accurate diagnoses. In such cases, enhanced cooperation with hematopathology could lead to increased awareness, feasibly to a more accurate diagnoses (14).

In B cell lymphomas, involvement of the spleen is usually evident but marked splenomegaly as a main finding in FL is rare (4). Despite that, $41.9 \%$ of patients had splenomegaly, which is much higher than the results of Koiso et al. (14). Although BM involvement may be present in up to $50-60 \%$ of patients, it was seen in $40.5 \%$ of the patients in our study. Finally, in terms of B symptoms, our results were parallel with Federico et al. (11), 15\% of their patients exhibited B symptoms whereas $13.5 \%$ exhibited it in ours.

Both for Hodgkin's and non-Hodgkin's lymphomas, the Ann Arbor staging system is used for staging the disease. The designations for the four different stages (I-IV) are A, B, E, and S, which reflect the patient's symptomatic findings. In our study, 5 (11.6\%) patients were in stages I-II and $31(72.1 \%)$ patients were in stages III-IV. Likewise, Federico et al. (11) had 250 (25\%) patients in stages I-II and 737 (75\%) of their patients were in stages III-IV.

In 2004, the FLIPI was assembled as a prognostic tool to facilitate estimating patients' overall survival based on their clinical information (15). Five different parameters are used; age (>60 vs $<60$ years), Ann Arbor stages (III-IV vs I-II), anemia (hemoglobin $<120 \mathrm{vs} \geq 120 \mathrm{~g} / \mathrm{L}$ ), number of $\mathrm{LN}$ area involved ( $>4 \mathrm{vs} \leq 4$ ), and serum LDH levels (elevated vs normal) (15). A point is granted when the answer is the first one out of the two choices cited above. Classification is made based on points acquired, 1 point is the low-risk group, which equals $70 \%, 2$ points is the intermediate-risk group, which equals $50 \%$, and 3 or more points means high risk, which equals $35 \%$ of the patients that had 10 -years of overall survival. In comparison with 51 months in Frederico et al. (11) study, findings regarding total follow-up of patients after their initial diagnosis were around 50 months in our study.
Standard initial treatment for symptomatic advanced indolent NHL is a rituximab-based immunochemotherapy regimen. Rituximab with cyclophosphamide, doxorubicin, vincristine, and prednisone (R-CHOP) chemotherapy has shown prominent results in progression-free survival, in addition, rituximab with cyclophosphamide, vincristine, and prednisone (R-CVP) regimen demonstrated an improvement in overall survival of patients (16, 17). Yet, results in BRIGHT study elucidated how bendamustine and rituximab combination was non-inferior to standard treatment regimen (18). A study previously discussed, conducted in Turkey by Kahraman et al. (12), used four different methods as first-line treatment: watch and wait, radiotherapy, chemotherapy, and rituximab. Percentages of their administrations were sequentially $7.1 \%$, $14.3 \%, 78.6 \%$, and $40 \%$. Demonstrating similar results, our study also had watch and wait (18.6\%), chemotherapy: R-CHOP, R-CVP (81.4\%), rituximab (20.9\%), and radiotherapy (2.3\%). Moreover, Obinutuzumab, lenalidomide, and bendamustine combination and rituximab with ifosfamide, carboplatin, and etoposide phosphate were used for the second-line treatment in our study.

In this retrospective study, the patient population was one of our main limitations while comparing our results with current literature. Our study also lacked some of the patients' information regarding their total follow-up, Ann Arbor stages, B symptoms, $\mathrm{BM}$ involvements, $\mathrm{LN}$ involvements, and treatment method due to missing data in patients' files.

In conclusion, despite being the most common NHL, FL usually ends up getting diagnosed at an advanced stage of the disease, presenting with incidentally noticed painless lymphadenopathy following a painless clinical course. This study aimed to make a contribution to current literature on the subject of demographic features, disease characteristics, total-follow up, and treatment methods. Furthermore, based on the evidence we have reviewed, a clear gap between patients in developed and developing countries can be observed in the diagnosis of FL. To overcome this hurdle, enhanced cooperation with hematopathology could lead to increased awareness, enabling physicians to make a more accurate diagnosis. Thus, further studies are required to fully apprehend the epidemiology of FL patients in Turkey.

Ethics Committee Approval: This retrospective study was approved by the Scientific Research Ethics Committee of Trakya University School of Medicine (Protocol Code: TUTF-BAEK2020/339).

Informed Consent: Informed consent was obtained from all subjects.

Conflict of Interest: The authors declared no conflict of interest.

Author contributions: Concept: FEA, BK, FOŞ, MYA, İ, EC, UD, HOK. Supervision: FEA, BK, FOŞ, MYA, İ, EC, UD, HOK. Resources: FEA, BK, FOŞ, MYA, İS, EC, UD, HOK. Materials: FEA, BK, FOŞ, MYA, İS, EC, UD, HOK. Data collection and/or processing: FEA, BK, FOŞ, MYA, İS, EC, UD, HOK. Analysis and/or Interpretation: FEA, BK, FOŞ, MYA, İS, EC, UD, HOK. Literature Search: FEA, BK, FOŞ, MYA, İS, EC, UD, HOK. Writing Manuscript: FEA, BK, FOŞ, MYA, İS, EC, UD, HOK. Critical Review: FEA, BK, FOŞ, MYA, İS, EC, UD, HOK.

Financial disclosure: The authors declared that this study received no financial support.

Editor-in-chief's Note: Five of the authors of this article, Fatih Erkan Akay, Beliz Koçyiğit, Fevzi Oktay Şişman, Mert Yücel Ayrık, Elif Cengiz are members of the editorial board of Turkish Medical Student Journal. However, they did not take place in any stage on the editorial decision of the manuscript. The editors who evaluated this manuscript are from other institutions. 


\section{REFERENCES}

1. Galaznik A, Huelin R, Stokes M et al. Systematic review of therapy used in relapsed or refractory diffuse large B-cell lymphoma and follicular lymphoma. Future Sci OA 2018;4(7):322.

2. Swerdlow SH, Campo E, Pileri SA et al. The 2016 revision of the World Health Organization classification of lymphoid neoplasms. Blood 2016;127(20):2375-90.

3. Dada R. Diagnosis and management of follicular lymphoma: a comprehensive review. Eur J Haematol 2019;103(3):152-63.

4. Project TN-HsLC. A clinical evaluation of the International Lymphoma Study Group classification of non-Hodgkin's lymphoma. Blood 1997;89(11):3909-18.

5. Agopian J, Navarro JM, Gac AC et al. Agricultural pesticide exposure and the molecular connection to lymphomagenesis. J Exp Med 2009;206(7):1473-83.

6. Freedman A, Jacobsen E. Follicular lymphoma: 2020 update on diagnosis and management. Am J Hematol 2020;95(3):316-27.

7. Kridel R, Sehn LH, Gascoyne RD. Pathogenesis of follicular lymphoma. J Clin Invest 2012;122(10):3424-31.

8. Turkish Hematology Association. Lymphoma diagnosis and treatment guideline. 1.4th edition. Turkey; 2020.

9. Sugimoto T, Watanabe T. Follicular lymphoma: The role of the tumor microenvironment in prognosis. J Clin Exp Hematop 2016;56(1):1-19.

10. Becnel MR, Nastoupil LJ. Follicular lymphoma: Past, present, and future. Curr Treat Options Oncol 2018;19(7):32.
11. Federico M, Vitolo U, Zinzani PL et al. Prognosis of follicular lymphoma: a predictive model based on a retrospective analysis of 987 cases. Blood 2000;95(3):783-9.

12. Kahraman S, Katgı A, Pişkin Ö et al. Tek merkez deneyimi: folliküler lenfomal olgularımızın retrospektif değerlendirmesi. Dokuz Eylül Üniversitesi Tıp Dergisi 2012;26(1):21-6.

13. Carbone A, Roulland S, Gloghini A et al. Follicular lymphoma. Nat Rev Dis Primers 2019;5(1):83

14. Koiso H, Yokohama A, Mitsui $\mathrm{T}$ et al. Follicular lymphoma presenting with marked splenomegaly: Report of three cases. Acta Haematol 2012;128(1):47-52.

15. Relander T, Johnson NA, Farinha P et al. Prognostic factors in follicular lymphoma. J Clin Oncol 2010;28(17):2902-13.

16. Hiddemann W, Kneba M, Dreyling $M$ et al. Frontline therapy with rituximab added to the combination of cyclophosphamide, doxorubicin, vincristine, and prednisone (CHOP) significantly improves the outcome for patients with advanced-stage follicular lymphoma compared with therapy with $\mathrm{CHOP}$ alone: Results of a prospective randomized study of the German Low-Grade Lymphoma Study Group. Blood 2005;106(12):3725-32.

17. Marcus R, Imrie K, Belch A et al. CVP chemotherapy plus rituximab compared with CVP as first-line treatment for advanced follicular lymphoma. Blood 2005;105(4):1417-23.

18. Flinn IW, van der Jagt R, Kahl BS et al. Randomized trial of bendamustine-rituximab or R-CHOP/R-CVP in first-line treatment of indolent NHL or MCL: The BRIGHT study. Blood 2014;123(19):2944-52. 